\title{
INFRARED IMAGES OF MERGING GALAXIES
}

\author{
G. S. Wright ${ }^{1}$, P. A. James², R. D. Joseph², I. S. McLean ${ }^{8}$, and R. Doyon 4
}

1) Joint Astronomy Centre, 665 Komohana Street, Hilo, HI96720.

2) Institute for Astronomy, 2680 Woodlawn Drive, Honolulu, HI96822.

3) Dept. of Astronomy and Physics, UCLA, 405 Hilgard Ave., Los Angeles, CA90024.

4) Blackett Laboratory, Imperial College, London, SW7 2BZ.

\section{INTRODUCTION}

Infrared imaging of interacting gnlaxies is especially interesting because their optical appearance is often so chaotic due to extinction by dust and emission from star formation regions, that it is impossible to locate the nuclei or determine the true stellar distribution. However, at near-infrared wavelengths extinction is considerably reduced, and most of the flux from galaxies originates from red giant stars that comprise the dominant stellar component by mass. Thus near infrared images offer the opportunity to study directly components of galactic structure which are otherwise inaccessible. Such images may ultimately provide the framework in which to understand the activity taking place in many of the mergers with high IRAS luminosities.

\section{MULTIPLE NUCLEI}

Infrared imaging is a powerful tool for the identification of galactic nuclei, since the nuclear regions of many galaxies are often too heavily enshrouded by dust for optical images to show the underlying structure. A well known example of this is Arp220, where the two optical maxima are due to a dust lane which bisects the galaxy and neither of them are associated with galaxy nuclei. We have obtained $\mathrm{K}(2.2 \mu \mathrm{m})$ images of two galaxies, Mkn788 and IRAS0857+3912 which have previously been described as the result of galaxy mergers on the basis of two equally bright optical peaks. Our image of Mkn788 (Joseph et al. 1988) shows that the western source is much more sharply peaked and has twice the integrated luminosity of the other. The brightest infrared source is coincident with the VLA source (Kollatschny et al. 1986), suggesting that it is the true nucleus of Mkn788. The broader, lower intensity K source which corresponds to the other "optical nucleus" could be interpreted as a giant extra-nuclear HII region similar to the jumbo HII region in NGC3310 (cf. Telesco \& Gatley 1984). In IRAS0857+3912 we find a similar result. The optical image presented by Sanders et al. (1988) shows two almost equally bright optical lobes, whereas in our $\mathrm{K}$ image the north-western source has almost an order of magnitude higher peak surface brightness than its neighbour. This extremely red source is quite possibly the nucleus of the IRAS galaxy. However, a third very red source, which is barely apparent even at $J(1.25 \mu m)$ is also clearly detected in the $\mathrm{K}$ image of this very disturbed galaxy. 
Infrared images have also been useful in identifying double structures in the nuclei of interacting galaxies which have not even been hinted at by optical observations. A striking example of this is given by the $\mathrm{K}$ images of Arp220. Graham et al. (1990) have used high resolution imaging to show that it has a double nucleus coincident with the radio sources in the middle of the dust lane.

The results discussed above suggest that caution should be applied in the identification of optical bright spots as multiple nuclei in the absence of other evidence. They also illustrate the advantages of using infrared imaging to study the underlying structure in merging galaxies.

\section{SURFACE PHOTOMETRY}

We have begun a programme to take near infrared images of galaxies which are believed to be mergers of disk galaxies because they have tidal tails and filaments. In many of these the merger is thought to have induced exceptionally luminous infrared emission (cf. Joseph and Wright 1985, Sanders et al. 1988). Although the optical images of the galaxies show spectacular dust lanes and filaments, the $\mathrm{K}$ images all have a very smooth distribution of light with an apparently single nucleus. Several authors (e.g. White 1979, Farouki \& Shapiro 1982, Barnes 1989) have predicted that over a period of $\sim 10^{\ominus}$ yrs mergers should produce a mass distribution indistinguishable from that of an elliptical galaxy, based on $\mathrm{n}$-body simulations of merging disk galaxies. Observational evidence in support of this idea is presented by Schweizer (1982), who showed that the V-light profile of NGC7252 is indeed well fitted by the $r^{1 / 4}$ de Vaucouleurs law. We have used the $\mathrm{K}$ images to test the hypothesis that other mergers are also developing elliptical-like light profiles.

Figure 1a shows profiles for Arp 220 and NGC 2623, in which K surface brightness within elliptical annuli is plotted against radius ${ }^{1 / 4}$. The characteristic de Vaucouleurs law for elliptical galaxies is a straight line on this plot. The lines shown were obtained by a least-squares fit to all points except for the inner two, which are artificially reduced due to seeing and decentering effects. The lines are clearly a good fit to the data over a range of about 4 magnitudes and linear scales of about $5 \mathrm{kpc}$ in radius, using $H_{0}=50 \mathrm{~km} \mathrm{~s}^{-1} \mathrm{Mpc}^{-1}$. This similarity with ellipticals is strengthened by the half-light scale lengths for the two mergers. The effective radius containing half the $\mathrm{K}$ light is $1.6 \mathrm{kpc}$ for NGC2623, and $3.4 \mathrm{kpc}$ for Arp220, consistent with published values for ellipticals (Kormendy 1982). Unfortunately the limited area covered by these data makes it impossible to rule out absolutely the existence of a more extended disk. We nevertheless conclude, on the basis of our present data, that Arp220 and NGC2623 have stellar light profiles which closely resemble those of elliptical galaxies.

$\mathrm{K}$ images for the remaining four galaxies show light profles which are not those of spiral or elliptical galaxies. Figure 1b shows examples of the kinds of deviation from simple light profles which we have found. IC883 (shown) and NGC4194 are well fitted by an $\mathrm{r}^{1 / 4}$ law beyond approximately $1 \mathrm{kpc}$, but the profile is significantly flattened within this radius. NGC6240 and NGC6052 (shown) differ from 
ellipticals or spirals over the entire surface brightness range. It is probably significant that these four galaxies all have nuclei substantially offset from the centre of the outer isophotes. The fact that the light profiles of Arp220 and NGC2623 are a much better fit to an $\mathrm{x}^{1 / 4}$ law than the other galaxies we have imaged so far suggests that Arp220 and NGC2623 may be the most dynamically evolved of the merger remnants associated with IRAS sources. In the case of Arp220 it is tempting to speculate that its stage of dynamical evolution is related to other suggestions that Arp220 is one of the "oldest" of the IR luminous mergers, based on scenarios for the evolution of the luminosity source.
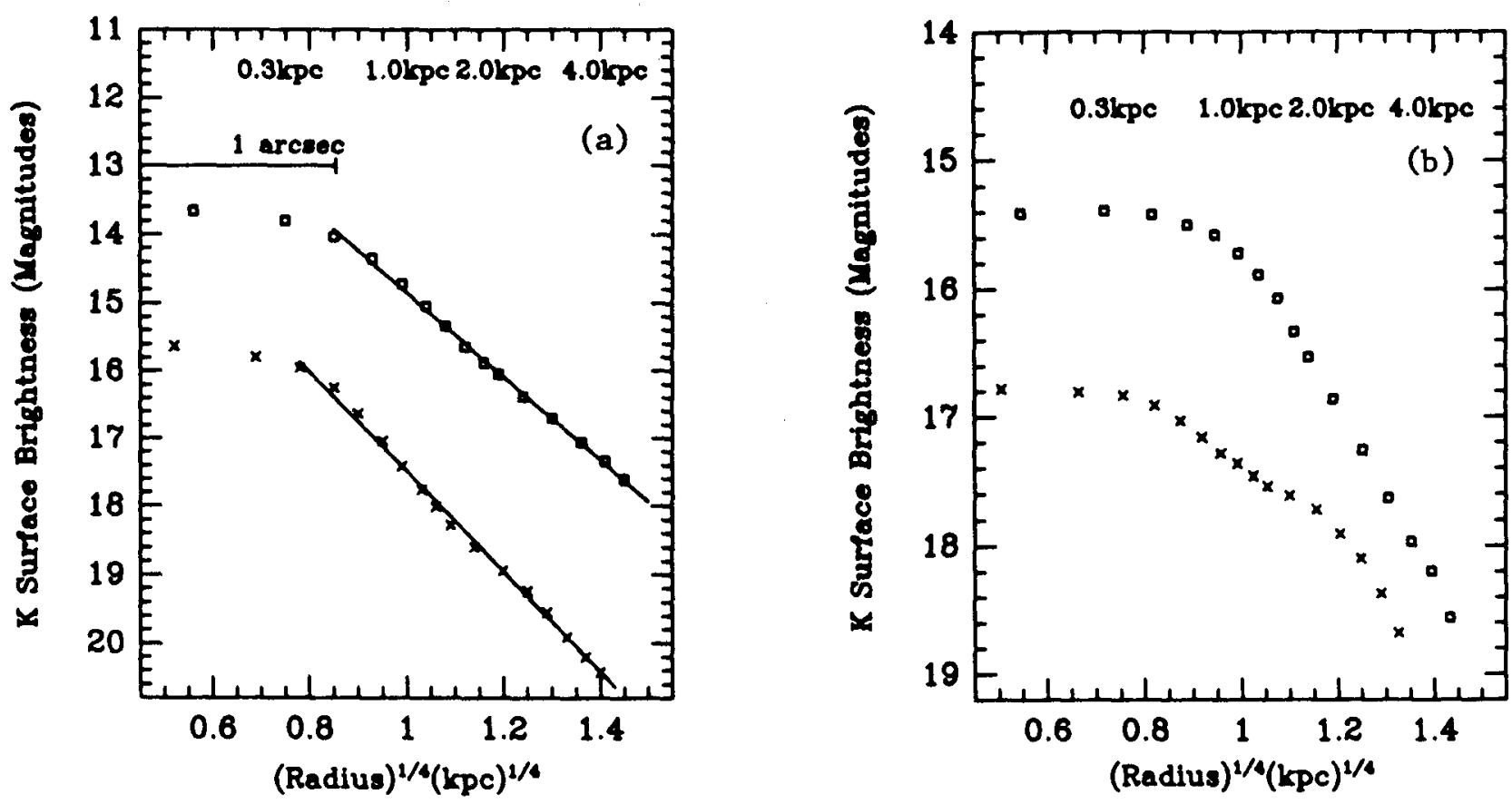

Figure 1 K surface brightness (mag/arcsec ${ }^{2}$ ) plotted vs. the fourth root of the radius for a) Arp220 (a) and NGC2623 (x) and b) IC883 (a) and NGC6052 (x). The plot for NGC2623 has been offset by +2 magnitudes for clarity. The scale along the top of both plots is radius in kpc, assuming $H_{0}=50 \mathrm{~km} \mathrm{~s}^{-1}$ $\mathrm{Mpc}^{-1}$.

While Arp220 and NGC2623 may have developed the mass distribution of an elliptical galaxy, they still have much more gas and dust. If merger remnants are actually to evolve into elliptical galaxies there must be some mechanism which removes the interstellar material. The activity which powers the luminous IR emission of these galaxies may play a key role in this process. For example, Graham et al. (1984) argue that a consequence of starbursts is a sufficiently high supernova rate to severely deplete the gas. 
Similarly it has been suggested (eg. Carlberg 1989, Efstathiou \& Rees 1988) that a quasar-like active nucleus could consume the gas in less than a Hubble time. We suggest therefore that the high IRAS luminosities of merging galaxies provide a clue to the means by which they could become as gas free as ellipticals.

\section{CONCLUSIONS}

Near infrared images are a much better way to investigate the existence of multiple nuclei than optical images because extinction and the emission from hot young stars are much reduced at $2.2 \mu \mathrm{m}$. Our images of Mkn 788 and IRAS $0857+3912$ are strikingly different from optical images in that only one nucleus clearly dominates in the near-IR. This suggests that in the absence of other evidence for interaction care should be taken in classifying potential mergers on the basis of nuclear structure in optical images alone.

$2.2 \mu \mathrm{m}$ surface photometry of several high luminosity mergers of disk galaxies has been used to test the idea that they may be evolving into ellipticals. Arp220 and NGC2623 obey the characteristic de Vaucouleurs law of ellipticals at $2.2 \mu m$ over several kpe, contrary to the impression given by optical images. We suggest that this provides support for the idea that mergers will evolve into elliptical-like objects. It is clear that the relation between degree of relaxation and other evolutionary processes in mergers is a very important question. By obtaining infrared images of a larger sample of mergers going to fainter isophotes we plan a more definitive study of the evolution of merger remnants with high IRAS luminosities.

\section{REFERENCES}

Barnes, J. E. 1989 Nature 338, 123.

Carlberg, R. G. 1989 Astrophys. J. preprint

Efstathiou, G. \& Rees, M.J. 1988 Mon. Not. R. astr. Soc. 230, 5p.

Farouki, R. T. \& Shapiro, S. L. 1982 Astrophys. J. 259, 103.

Graham, J. R., Wright, G. S., Meikle, W. P. S., Joseph, R. D., \& Bode, M. F. 1984 Nature 310, 213.

Graham, J. R., Carico, D. P., Matthews, K., Neugebauer, G., Soifer, B. T. \& T. D. Wilson 1990

Astrophys. J. Lett. preprint

Joseph, R. D. \& Wright, G. S. 1985 Mon. Not. R. astr. Soc. 214, 87.

Joseph, R. D., Wright, G. S., James, P. A., \& McLean, I. S. 1988 Mon. Not. R. astr. Soc. 232, 7p.

Kollatschny, W., Netrer, H. \& Frike, K. J., 1986 Astr. Astrophys., 163, 31.

Kormendy, J. 1982 Morphology and Dynamics of Galaxies ed. Martinet, L. SAAS-FEE 115.

Sanders, D. B., Soifer, B. T., Elias, J. H., Madore, B. F., Matthews, K., Neugebauer, G. \& Scoville, N. Z. 1988 Astrophys. J. 325, 74. 
Schweizer, F. 1982 Astrophys. J. 252, 455.

Telesco, C. M., Decher, R. \& Gatley, I., 1985. Astrophys. J. 299, 896.

Telesco, C. M. \& Gatley, I., 1984 Astrophys. J. 284, 557.

White, S. D. M. 1979 Mon. Not. R. astr. Soc. 189, 831. 


\section{DISCUSSION}

Lamb: I suggest a mechanism for the production of a large HII region away from the nucleus of an interacting galaxy. If two disk galaxies with gas collide and overlap for awhile, it is possible under some circumstances for the angular momentum to cancel in regions away from the nuclei. If this region contains gas, the collision may leave behind a region which is a prime candidate for star formation. Is it possible that there is another galaxy close to the one you are investigating which might have produced such an HII region?

Wright: Yes, this emphasizes why a single optical photograph does not necessarily show two nuclei, if it has two bright sources. Other information, (which may be a $K$ image) is needed to establish a merger. In fact, for one of the images I showed, notice that there is a third source which may be a companion galaxy on the $\mathrm{K}$ image.

Noreau: What kind of dynamical evidence do we have that Arp 220 is actually merging? I mean spectra from which we can derive Doppler-shift information.

Wright: Dr. Norris drew attention to spectra showing line widths in excess of $1500 \mathrm{~km} / \mathrm{s}$ and to the large velocities seen in molecular gas.

Khachikian: Do you think that superassociations can be the nuclei of galaxies?

Wright: Yes, an HII region can be at the nucleus of a galaxy, where by nucleus I mean the peak of the stellar density. But optical images showing two bright knots in a galaxy are not sufficient on their own to show that the galaxy has two nuclei and therefore must be a merger. Other data must also be used.

Heckman: What is the evidence that either the "nuclear" or "extra-nuclear" K-band light originates from an old stellar population? If the near-IR is produced by young stars, its $r^{1 / 4}$ surface brightness law is not readily explained by standard merger simulation. The two-color diagrams you showed suggested that the near-IR continuum was not from a normal old stellar population.

Wright: In general, the $\mathrm{K}$ band light off the nucleus does trace the old stellar population, and JHK colors are consistent with this. This need not be so on the nucleus (central 1-2 kpc). Near-IR colors can show a contribution to the K-light due to hot dust, non-thermal emission, young stars, etc. The figure I showed for IRAS $08572+3912$, showed nuclear colors indicating components that are not necessarily stars. 